\title{
Analysis of the transcriptome of CD4 T-Lymphocytes from young Non-Obese Diabetic (NOD) mice suggests a key role for cytokines and apoptosis
}

Dorothy N Kakoola ${ }^{1,2}$, Nataliya I Lenchik ${ }^{1}$, Anita Curcio-Brint ${ }^{1}$ and Ivan C Gerling*1

\author{
Address: ${ }^{1}$ Department of Medicine, University of Tennessee Health Science Center, Memphis, TN 38104, USA and ${ }^{2}$ Research Service, Veterans \\ Affairs Medical Center, Memphis, TN 38104, USA \\ Email: Ivan C Gerling* - igerling@utmem.edu \\ * Corresponding author
}

from UT-ORNL-KBRIN Bioinformatics Summit 2008

Cadiz, KY, USA. 28-30 March 2008

Published: 8 July 2008

BMC Bioinformatics 2008, 9(Suppl 7):PI6 doi:I0.1 186/I47I-2105-9-S7-PI6

This abstract is available from: http://www.biomedcentral.com//47I-2105/9/S7/PI6

(c) 2008 Kakoola et al; licensee BioMed Central Ltd.

\section{Background}

The earliest histological signs of autoimmunity in female NOD mice occur at approximately 4 weeks of age. We hypothesized that defects associated with these events may be evident in the molecular signatures of spleen leukocytes. To gain a detailed insight into these early molecular defects we analyzed the transcriptomes of CD4 Tlymphocytes, the predominant leukocyte subset involved in autoimmune diabetes.

\section{Methods}

Using a magnetic separation system (Miltenyi Biotec), we isolated CD4 T-lymphocytes from spleen leukocytes of female NOD mice, and two control strains, NOR and $\mathrm{C} 57 \mathrm{Bl} / 6$, at 4 weeks of age ( $\mathrm{n}=5$ for each strain). CD4 Tlymphocyte transcriptomes were characterized on Affymetrix Mouse 430_2 expression arrays probing 39,000 mouse genes/ESTs. Gene lists produced from statistical analyses were analyzed using Ingenuity Pathway Analysis http://www.ingenuity.com.

\section{Results}

Analysis by 1-way ANOVA, with a Benjamin-Hochberg Multiple Test Correction (MTC), defined 581 probesets (genes) with highly significant expression differences between strains $(\mathrm{p}<0.005)$. Hierarchical clustering of these 581 probesets revealed that 65 probesets were differ- entially expressed in NOD mice relative to both control strains. Nine were upregulated in NOD mice while 56 were downregulated. Twenty-six of these 65 probesets created a single large molecular network in pathway analysis. The network clustered around pro-inflammatory cytokines (TNF- $\alpha$ and IL-6), Th1-Th2 regulatory cytokines (IFN- $\gamma$ and IL-4), apoptosis regulating genes (BCL2L1, EGF and TP53), and the iNOS gene. Furthermore, analysis by an anchored clustering method revealed an additional 27 probesets whose expression patterns were similar to that of the above-mentioned 65 probesets although at a lower statistical level ( $p<0.05$, no MTC). Nine of these 27 probesets created an Ingenuity Pathway network centered on IFN- $\gamma$, TP53 and EGF, further delineating the importance of these genes in expression abnormalities in CD4 Tlymphocytes from NOD mice.

We conclude that molecular systems abnormalities (biomarkers) associated with inflammation (TNF- $\alpha$, IL6), Th1-Th2 differentiation (IFN- $\gamma$, IL-4), apoptosis (BCL2L1, EGF, TP53) and nitric oxide (iNOS) are evident at 4 weeks of age in CD4 T-lymphocytes of female NOD mice.

\section{Acknowledgements}

This work was supported by a research supplement grant from the National Institute of Diabetes \& Digestive \& Kidney Diseases, DK062I 03. 